\title{
Novel High Bandwidth Metamaterial Antenna for WIMAX and WPAN Applications in $\mathbf{C}$ and $\mathrm{X}$-band
}

\author{
${ }^{1}$ V.R.Anitha, ${ }^{2}$ P.Gnaneswari \\ ${ }^{1}$ Antenna Research Lab, Sree Vidyanikethan Engineering College, A Rangampet, Tirupati, Andhra Pradesh, India - \\ 517102. \\ ${ }^{2}$ Sree Vidyanikethan Engineering College, A Rangampet, Tirupati, A.P, India - 517102. \\ Email: anithavr@ieee.org,gnanipavan@gmail.com
}

Received: 06th April 2020, Accepted: 05th May 2020, Published: 30th June 2020

\begin{abstract}
The performance of patch antenna loaded with H-Shape SRR with aperture feeding technique has been described in this paper. It has been proposed in an H-shaped antenna with metamaterial loading which provides a conceptual route for the implementation of small resonant antennas. We Crafted a rectangular microstrip patch antenna that has the relative permittivity 2.2 and the electrical loss tangent of 0.0009 . The new antenna has wider bandwidth and increased performance in radiation. The novel antenna has compact dimensions of $4.2 \mathrm{~mm}$ X $5.2 \mathrm{~mm}$. The simulated parameters like return loss, band width, and directivity have been done for aperture feeding at different frequencies.
\end{abstract}

\section{Keywords}

Split Ring Resonator, Metamaterial, Patch Antenna, Aperture-coupled Feeding, HFSS Simulation Software, WLAN

\section{Introduction}

In modern days, wireless applications has lot of demand like WLAN (wireless local area network)[1], WPAN (wireless personal area network). The main constraints in wireless applications are small in size, low cost and easy to fabricate. As the technology increases, the requirement of antenna to resonate at multiple frequencies increases. The above requirements should be satisfied by the microstrip patch antenna. Patch antenna can be mounted on a flat surface because it is one type of radio antenna. The patch has many advantages of low profile, low cost, capable of double and triples frequency operation, easy to fabricate, comfortable on curved surface and easily integrated with microwave integrated circuits [2]. The main limitations of patch antenna are poor power handling capacity, narrow bandwidth, and low cross polarization [3]. Most of these drawbacks are overcome by new structure like metamaterials etc.

In Greek, Meta means beyond. Metamaterials are engineered materials that have unique property not found in nature due to their periodic arrangement and design. First artificial metamaterial was theoretically designed by Victor veselago [4] in 1968. But at that time practically no material exhibit negative permittivity and permeability. The first artificial metamaterial in millennium [5] have been fabricated by Smith and his group. In 1999, Dr. John pendry showed practical method of making metamaterial by using SRR structure. The structure of metamaterial can also miniaturizes the size of patch antenna, increases the gain and sometimes achieves better return loss.

In 2000, Smith et al. built a split ring resonator whose permeability is negative. Single cell split ring resonator has pair of enclosed loops with split on opposite end. The splits are exactly $180^{\circ}$ apart from each other and the loops are made up of non-magnetic metal like copper. Due to split on the rings, the arrangement will hold up resonant wavelength much superior than the diameter of ring this would not occur in closed loop structures. The difference between the inner and outer rings acts as a capacitor and the rings themselves serve as an inductor resulting in LC resonant circuit. Many types of split-ring resonators were developed by few researchers. They are Edge-coupled SRR (EC SRR), Broad-side coupled SRR (BC-SRR), Non-bianistropic SRR (NB-SRR) and Spiral SRR. This article is an example of H-shaped edge coupled SRR (EC-SRR), which has two metal split rings printed on a microwave circuit board with break on each ring.

\section{H-Shape Split Ring Resonator (H-SRR) Design}

The H-shaped patch antenna is designed on the low-cost substratum FR-4. The antenna is fed by 50 coaxial impedance feed that can easily be mounted on a single PCB with other microwave circuits. This makes the antenna attractive with very limited space for emerging mobile applications. The patches are generally fed to symmetry along the center line and thus reduce the excitation of unwanted modes. Microstrip antennas are used at microwave and millimeter wave frequency $[3,7,8]$.

The performance of patch antenna is mainly affected by the design parameters of the SRR like split between the rings, number of split rings, shape and size of the rings. . Here, the work focuses on effect of H-shape SRR [9] on performance characteristics of patch antenna at different frequencies. The dimensions of H-shape SRR as shown in 
Fig. 1 are length of H- shape SRR is, $L_{s}=4.2 \mathrm{~mm}$; width of the SRR is $W_{S}=5.2 \mathrm{~mm}$ and gap between split rings $G_{r}$ $=0.3 \mathrm{~mm}$ and width of the ring is $W_{r}=1.1 \mathrm{~mm}$.

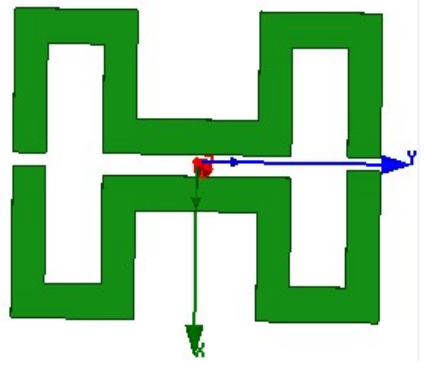

Fig. 1: Edge Coupled H-shape SRR

\section{Antenna Incorporated with Single H-shape SRR}

There are several feeding techniques to transmit electromagnetic energy to patch antenna. In order to improve the antenna impedance match the feeding place very important role. Different feeding techniques that are used in antennas are listed below, they are

1. Microstrip line feed

2. Coaxial feed

3. Aperture coupled feed

4. Proximity coupled feed

The most commonly used feeding technique is line and coaxial feeding. In this division we will concentrate on the effect of single H-shape SRR on the patch antenna at different frequencies by using aperture-coupled feeding.

\section{Aperture fed patch antenna loaded with H-shape SRR:}

When compared to the direct fed patch the aperture-coupled patch has more degree of freedom for the designer because of more design parameters. In aperture coupling, there are no sudden current discontinuities when compared to direct contact feed due to this reason the aperture coupled feed gives very accurate results when compared to direct feeding technique [10]. Therefore, this model is fairly simple, precise, and quick computational. Aperture coupled feed, in general form, has two substrates separated by ground plane. The patch is placed on one substrate, and other feed is placed, i.e. Separate patch and feed line by ground aircraft. Coupling is rendered through the slot between the patch and the feed line and is as shown in Fig.2. The different shapes that are used in aperture feed are rectangle, square, triangle, circular etc.

Because of two substrates, the key downside in aperture-coupled feed increases the volume of the antenna and also raises difficulties in manufacturing. Another disadvantage is the multi-layered substrate resulting in coupled- surface wave modes with a slot on the ground-plane. These modes cause skewed radiation patterns and also reduce the efficiency of the radiation [11].

In general, the slot is placed beneath the center of the patch resulting in lower cross polarization. The amount of coupling is determined according to the form, scale, and position of the opening. We can get better radiation pattern, small bandwidth and better gain without changing the resonant frequency by varying SRR location and feed line.

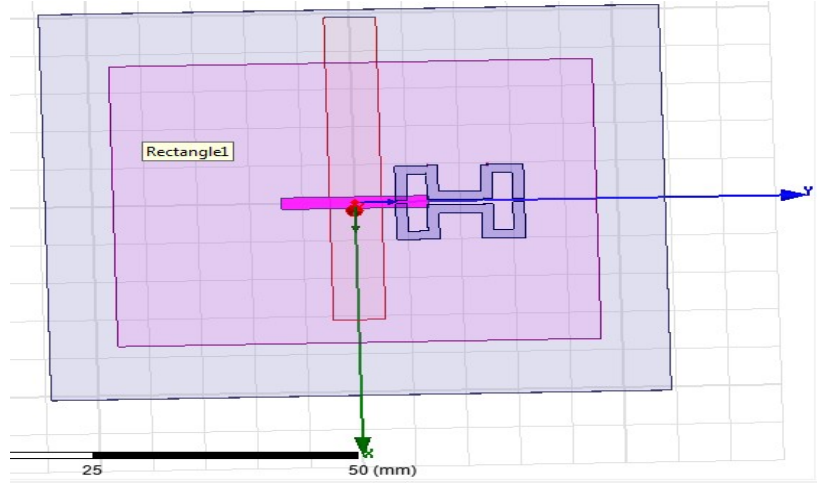

Fig. 2: The Incorporated H-Shape SRR onto Aperture fed Patch Antenna. 


\section{Results}

Simulated results from a full wave solver and analyzed. The efficiency characteristics of various frequencies such as $2.4 \mathrm{GHz}, 5.6 \mathrm{GHz}$ and $9.6 \mathrm{GHz}$ such as return loss, VSWR, gain, bandwidth, directionality, and radiation pattern are tabulated and shown in Table1.

\section{Results of Aperture-Coupled Patch Antenna Loaded with H- Shape Split Ring Resonator at 2.4 GHz:}

The return loss curve is shown in Fig. 3(a) for the rectangular patch antenna loaded with H-shape SRR using aperture feeding. S-band is used for wireless local area network (WLAN) applications to pick the resonant frequency of the proposed antenna. The dielectric material that can be used for designing of patch is RT Duroid 5880. The dimensions of the patch and dielectric substrate at resonant frequency $2.4 \mathrm{GHz}$ is $4 \mathrm{~cm} \times 4.8 \mathrm{~cm}, 5.1 \mathrm{~cm} \times 5.9 \mathrm{~cm}$, and the thickness of the substrate should be taken as $0.15748 \mathrm{~cm}$. At port 1 where the input is given to the patch antenna, $S_{11}$ gives the reflection coefficient and should not be less than $-10 \mathrm{~dB}$ for acceptable operation. The $\mathrm{S}_{11}$ is $-48.10 \mathrm{~dB}$ for an aperturecoupled $2.4 \mathrm{GHz}$ patch antenna. Due to placing of H-shape SRR into patch antenna leads to achieve multiple resonances at $4.8 \mathrm{GHz}$ and return loss at this frequency is $-17.54 \mathrm{~dB}$ and the simulated impedance band width of about $340 \mathrm{MHz}$ is achieved at $-3 \mathrm{~dB}$ reflection coefficient. The simulated parameters like VSWR, Gain, Directivity and radiation pattern are shown in Fig. 3.

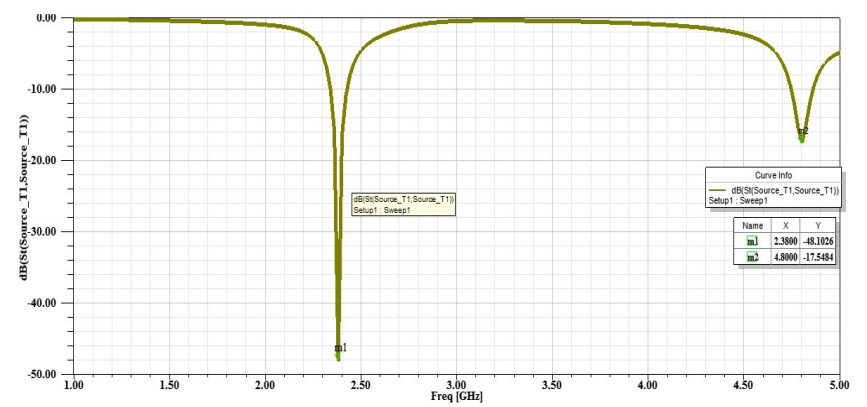

Fig. 3 (a): Reflection Coefficient of the Novel antenna at $2.4 \mathrm{GHz}$

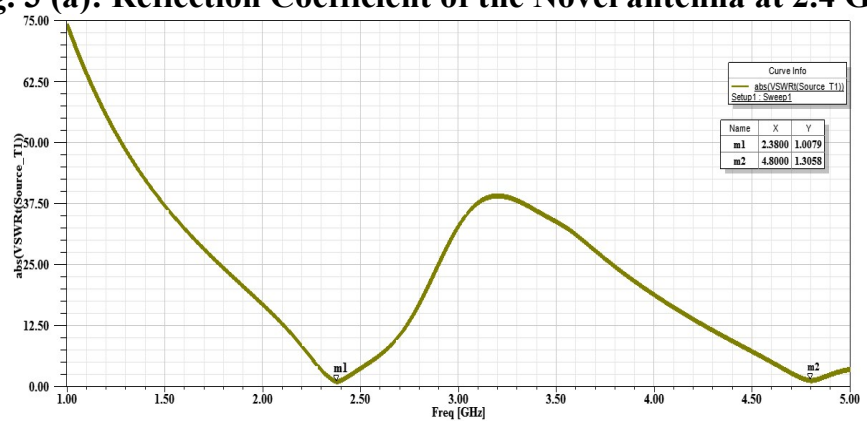

Fig. 3 (b): VSWR at $2.4 \mathrm{GHz}$

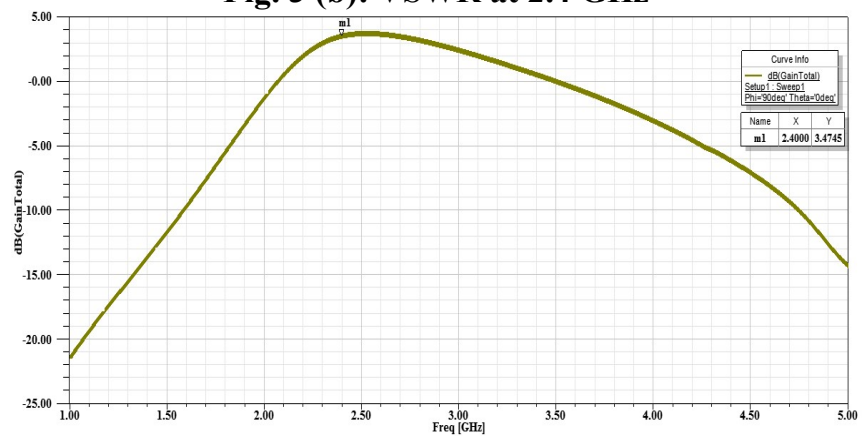

Fig. 3 (c): Gain at $2.4 \mathrm{GHz}$ 


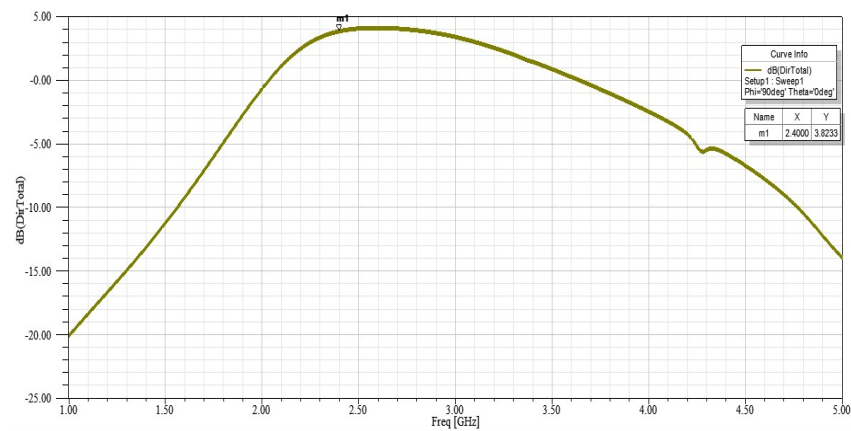

Fig. 3 (d): Directivity at $2.4 \mathrm{GHz}$

The graphical representation of the radiation pattern of the antenna as a function of space coordinates. The radiation pattern at S-band is shown in fig 4.1(e).

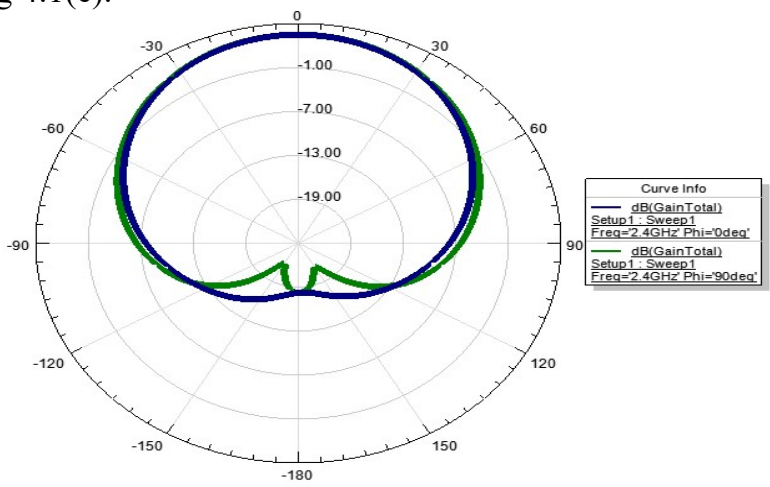

Fig. 3 (e): Radiation Pattern at 2.4 GHz and $\phi=0^{0}, 90^{0}$

3-D radiation pattern with gain $2.74 \mathrm{~dB}$ for proposed antenna at resonant frequency $2.4 \mathrm{GHz}$ is shown in Fig.3 (f).
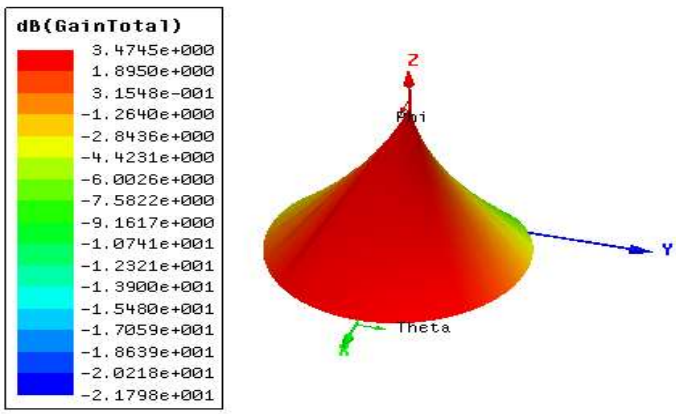

Fig. 3 (f): 3-D Radiation Plot at 2.4GHz

\section{Results of Aperture-Coupled Patch Antenna Loaded with H-Shape SRR at 5.6 GHz:}

For aperture coupled patch antenna equipped with H-shape Split Ring Resonator, the measurements of patch and ground plane are $1.7 \mathrm{~cm} 2.1 \mathrm{~cm}, 2.7 \mathrm{~cm} 3.1 \mathrm{~cm}$. In this, the proposed antenna's reflection coefficient $\mathrm{S}_{11}$ is $-41.63 \mathrm{~dB}$ at resonant frequency $5.6 \mathrm{GHz}$ as shown in Fig. 4(a) widely used for wireless applications such as Wi-Max. The resonant band width at $5.6 \mathrm{GHz}$ is $540 \mathrm{MHz}$. The performance characteristics such as VSWR, directivity and bandwidth are as shown in Fig.4. 


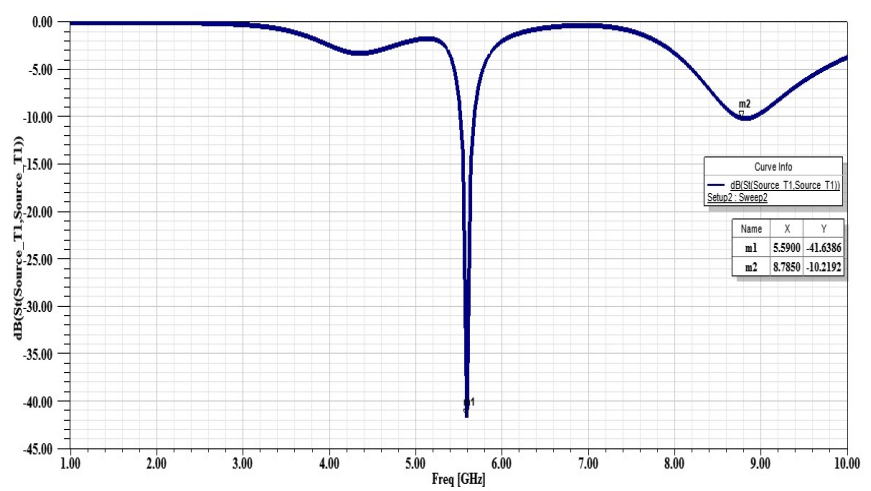

Fig.4 (a): Reflection Coefficient of the Proposed Antenna at 5.6 GHz

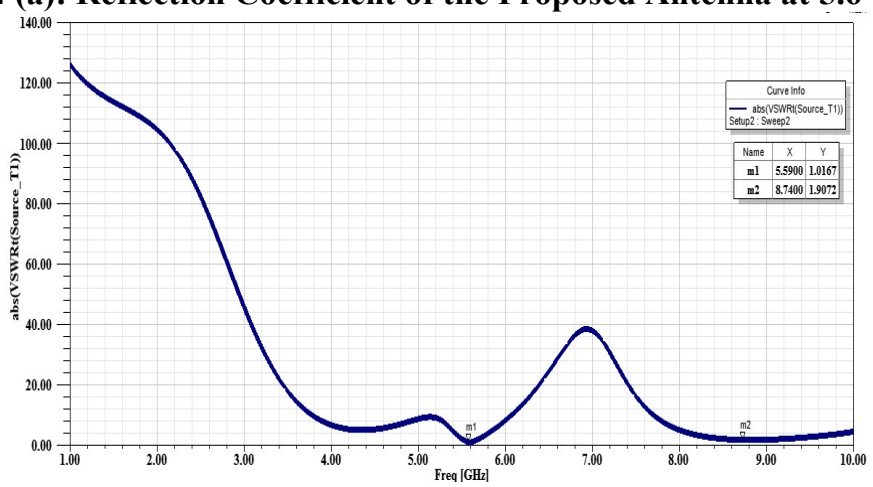

Fig.4 (b): VSWR at $5.6 \mathrm{GHz}$

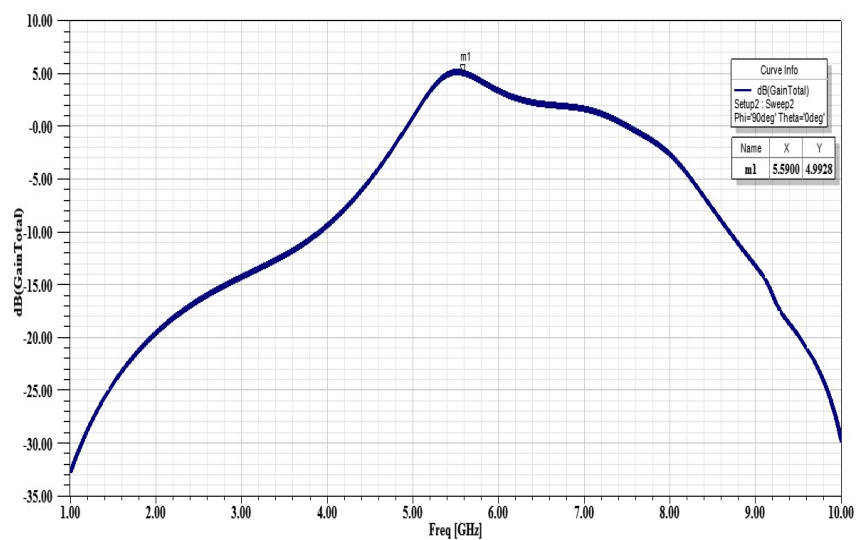

Fig.4 (c): Gain at $5.6 \mathrm{GHz}$

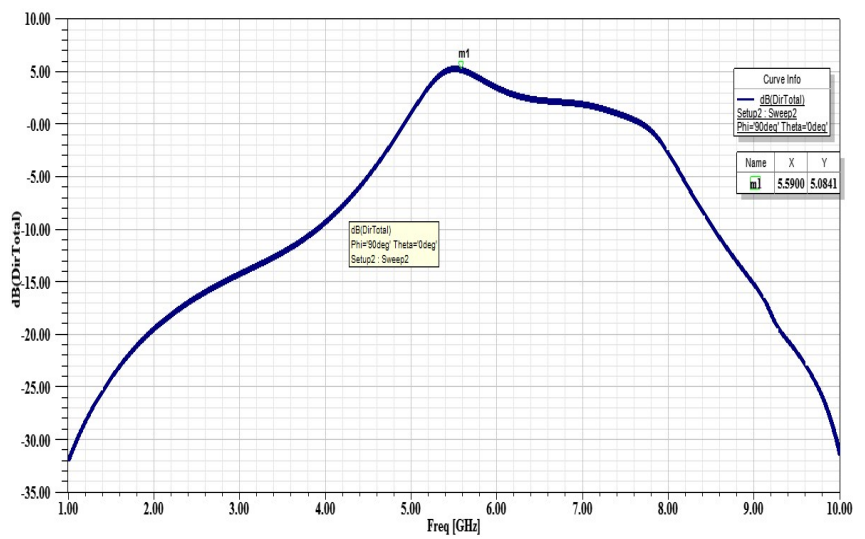

Fig.4 (d): Directivity at $5.6 \mathrm{GHz}$ 


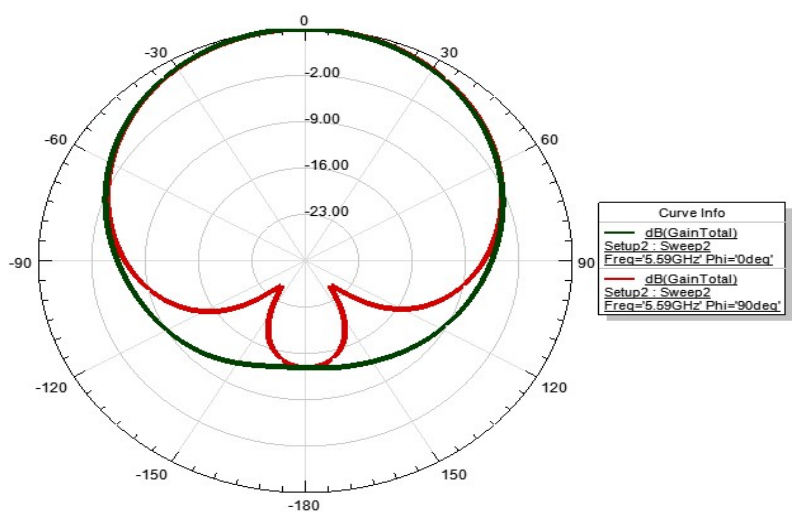

Fig.4 (e): Radiation Pattern at 5.6 GHz and $\phi=0^{0}, 90^{\circ}$
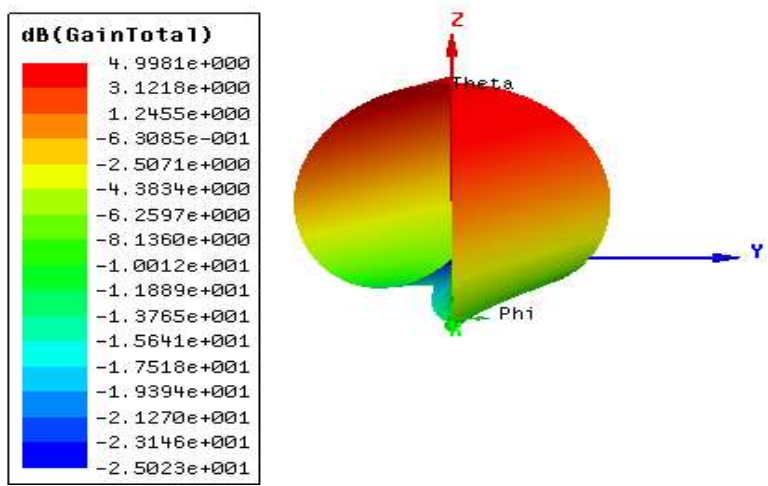

Fig.4 (f): 3-D Radiation Plot at 5.6 GHz

3. Results of Aperture-Coupled Patch Antenna Loaded with H-shape SRR at 9.5 GHz:

In this the proposed antenna's reflection coefficient $\mathrm{S} 11$ is $-40.92 \mathrm{~dB}$ at resonant frequency $9.5 \mathrm{GHz}$ as shown in Fig.5(a), which is widely used for wireless applications such as wireless personal area networks (WPANs). The resonant band width at $9.5 \mathrm{GHz}$ is $1650 \mathrm{MHz}$ (or) $1.65 \mathrm{GHz}$. The performance characteristics such as VSWR, gain, directivity and radiation pattern are shown in Fig. 5. Table.1 demonstrates comparison of the output characteristics of antenna loaded with H-shape SRR at different frequencies.

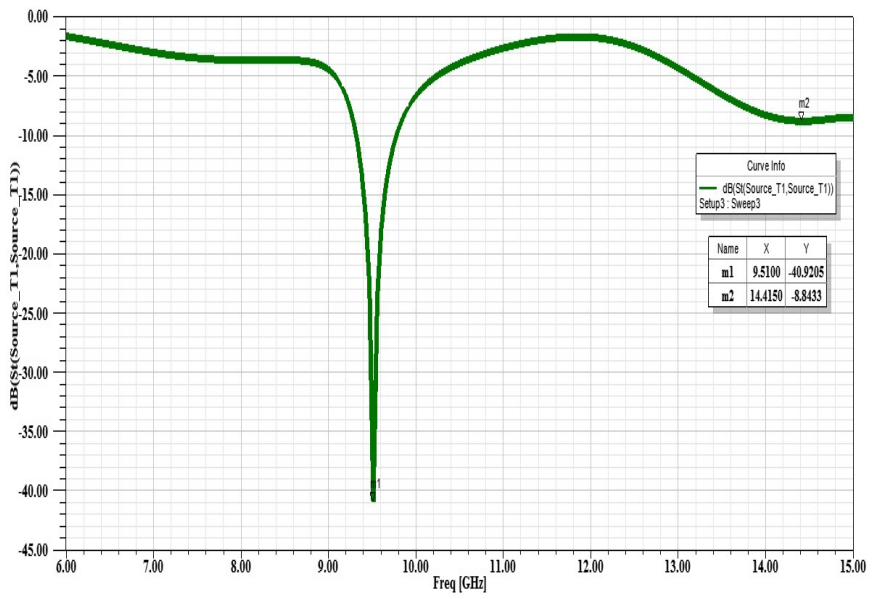

Fig. 5 (a): Reflection Coefficient at 9.5GHz 


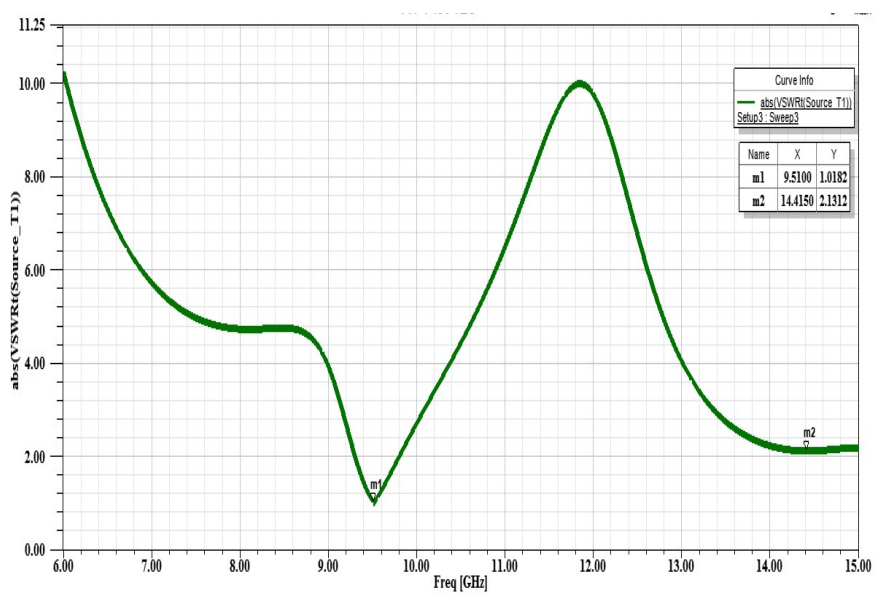

Fig. 5 (b): VSWR at 9.5GHZ

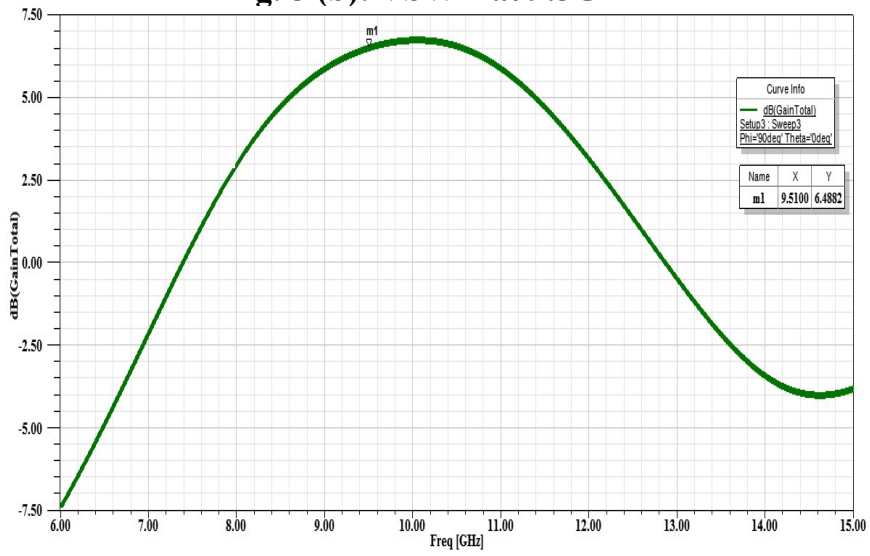

Fig. 5 (c): Gain at $9.5 \mathrm{GHz}$

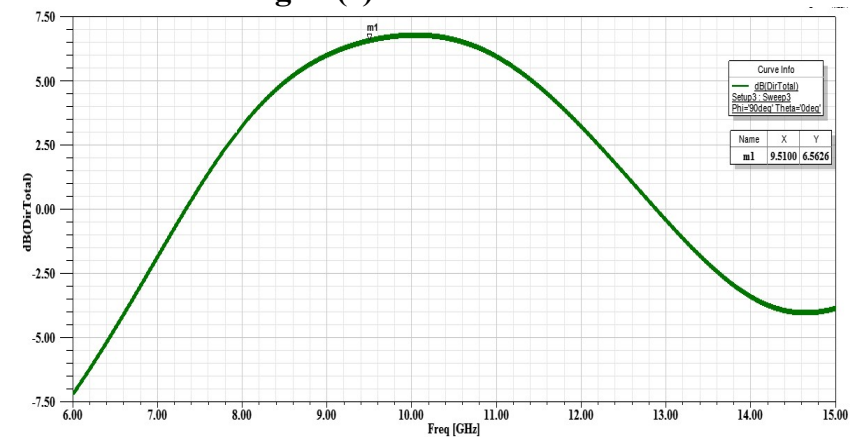

Fig. 5 (d): Directivity at $9.5 \mathrm{GHz}$

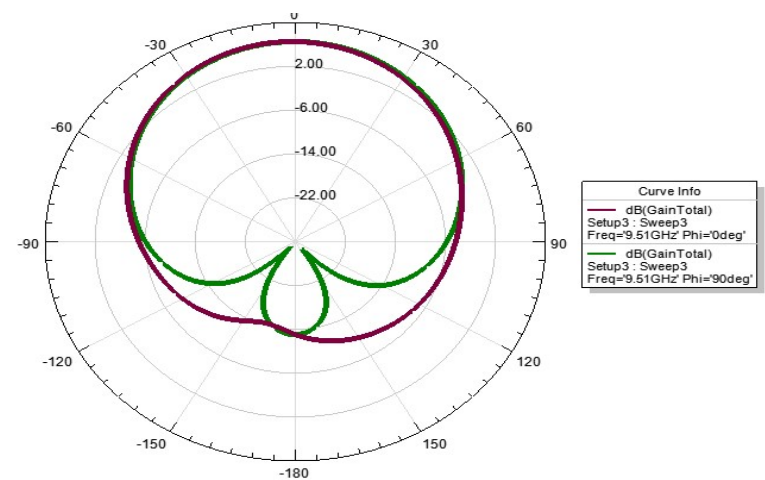

Fig. 5 (e): Radiation Pattern at $9.5 \mathrm{GHz}$ and $\phi=0^{0}, 9^{\circ}$ 


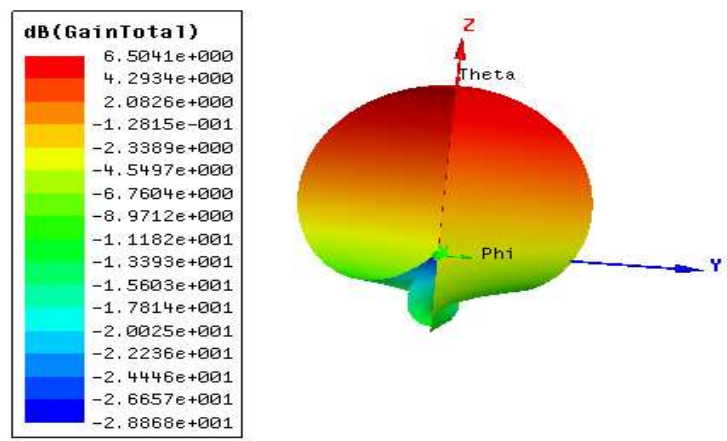

Fig. 5 (f): 3-D Radiation Plot at 9.5 GHz

Table 1: Coaxial Fed Patch Antenna Performance Characteristics Loaded at Different Frequencies with Hshape Split Ring Resonator.

\begin{tabular}{|c|c|c|c|}
\hline \multirow{2}{*}{ Parameter } & \multicolumn{3}{|c|}{ Resonant frequency $f_{0}$} \\
\cline { 2 - 4 } & $2.4 \mathrm{GHz}$ & $5.6 \mathrm{GHz}$ & $9.5 \mathrm{GHz}$ \\
\hline Return loss $(\mathrm{dB})$ & -48.10 & -41.63 & -40.92 \\
\hline VSWR & 1.007 & 1.01 & 1.01 \\
\hline Band Width (MHz) & 340 & 545 & $650 \mathrm{MHz}$ (or) $1.65 \mathrm{GHz}$ \\
\hline Gain (dB) & 3.47 & 5.0 & 6.49 \\
\hline Directivity (dB) & 3.82 & 5.08 & 6.56 \\
\hline
\end{tabular}

\section{Conclusion}

Patch antenna loaded with H-shape SRR for wireless applications such as WLAN, WI-MAX and WPAN using aperture-coupled is proposed. From the results, conclude that multiple resonances are obtained by loading H-shape SRR. This proposed microstrip patch antenna loaded with H-shape SRR enhanced the impedance band width and maximum band width achieved at $9.5 \mathrm{GHz}$ is $1.65 \mathrm{GHz}$, and provides good matching.

\section{References}

[1]Jaswinder Kaur,Rajesh Khanna,"Co-axial Fed Rectangular Microstrip Patch Antenna For 5.2GHz WLAN Application", universal Journal of Electrical and Electronic Engineering 1(3):94-98, 2013.

[2] C. A. Balanis, “Antenna Theory Analysis and Design”, John Wiley, Inc., New Jersey, 2005.

[3] D. M. Pozar and D. H. Schaubert, Microstrip Antennas: "The Analysis and Design of Microstrip Antenna and Arrays", IEEE Press, 1995.

[4] V. G. Veselago, "The Electromagnetic of Substances with Simultaneously Negative value of Permittivity and Permeability", Soviet Physics USPEKI, vol. 10, no. 4, pp.509-514,1968.

[5] D. R. Smith, W. J. Padilla, D. C. Vier, S. C. Nemat Nasser, S.Schultz, "Composite Medium with Simultaneously Negative Permeability and Permittivity", Phys. Rev. Lett., vol. 84, pp. 4184-4187, 2000.

[6] J. B. Pendry, A. J. Holden, D. J. Robbins, W. J. Stewart, "Magnetism From Conductors and Enhanced Nonlinear Phenomena",IEEE Trans. Microwave Theory Tech., vol. 47, pp. 2075-2084, 1999.

[7] R. Garg, P. Bhartia, I. Bahl, and A. Ittipiboon, "Microstrip Antenna Design Handbook," ArtechHouse, 2000.

[8] K. F. Lee, Ed., "Advances in Microstrip and Printed Antennas", John Wiley, 1997.

[9] Machac, J., Rytir, M., Protiva, P.; Zehentner, J., "A Double H-Shaped Resonator for an Isotropic ENG Metamaterial ," $38^{\text {th }}$ European Microwave Conference (EuMC 2008), pp. 547-550,2008.

[10] M.K.A. Rahim, Z.W Low, P.J Soh, A. Asrokin, M.H. Jamaluddin, and T.Masri, "Aperture Coupled Microstrip Antenna with Different Feed Sizes and Aperture Positions", 2006 International RF and Microwave Conference Proceedings, September 12-14,2006.

[11] L.C Godara, R. Waterhous, "Handbook of Antenna in Wireless Communication", CRC Press LLc,Florida, USA, Chap.6,p.p 5-10,2002. 